\title{
Random Error Characteristics and Evaluation Method of Optical Voltage Sensor
}

\author{
Cong $\operatorname{Lin}^{1,2^{*}}$, Min Cao ${ }^{1,2}$, Bo Li ${ }^{1,2}$, Qingchan $\mathrm{Liu}^{1,2}$, Quancong Zhu ${ }^{1,2}$ and Lihui Wang ${ }^{3}$ \\ ${ }^{1}$ Electric Power Research Institute of Yunnan Power Grid co. Ltd, Kunming 650217, China \\ ${ }^{2}$ Key Laboratory of Electric Power Measurement, China Southern Power Grid, Kunming, 650217, China \\ ${ }^{3}$ School of Instrument Science and Engineering, Southeast University, Nanjing, 210096, China \\ ${ }^{*}$ Corresponding author
}

\begin{abstract}
The random error characteristics of optical voltage sensors (OVS) directly affect the measurement accuracy. Sequence variance is a commonly used method to evaluate the characteristics of the OVS random error. However, sequence variance does not model the OVS noise itself and does not identify the noise coefficient in terms of OVS noise signature analysis. Based on sequence variance method, the improved sequential analysis of variance method is proposed to analyze the random error characteristics of OVS. After the OVS test data is processed by the improved sequential analysis of variance method, the least square fitting is performed, and the coefficients of each random error term are accurately solved. With the coefficients, the influencing factors are identified, and the corresponding error suppression measures are proposed. The experiments results demonstrate that the improved sequential analysis of variance method is different from the sequence variance method, the sequence variance method fluctuates and gradually increases when the correlation time is more than half of the total measurement duration, while the improved sequential analysis of variance method always remains relatively stable.
\end{abstract}

Keywords-optical voltage sensor; frequency domain stability component; random error; style; noise; evaluation

\section{INTRODUCTION}

Optical voltage transformers overcome the limitations of electromagnetic current transformers in terms of accuracy, dynamic range and DC measurement. Optical voltage transformers have a series of advantages with high measurement accuracy, large dynamic range, wide frequency response range and anti-electromagnetic interference and digital output[1]. The issue of random error characteristics faced by OVS has been one of the major constraints that limit the application of OVS in power system control, protection, measurement and metering. OVS random error mainly comes from light source, detection circuit, fiber ring, optical devices such as a series of time-varying noise[2-4]. After the signal processing part of the interference signal containing nonreciprocal phase filtering, conversion, signal processing, the characteristics of OVS output data mainly include random walk current noise, bias instability, random walk noise, rate ramp noise, quantization noise and sinusoidal noise.

Sequence variance is a universal method to characterize frequency stability and is widely used in the analysis of error characteristics of precision instruments. Relevant literatures have verified the feasibility of sequence variance noise modeling theory in OVS noise feature, however, the OVS noise has not been modeled and the noise coefficient has not been identified. In this paper, the improved sequential analysis of variance method is used to analyze the random error characteristics of OVS. Five types of random errors of OVS are analyzed, and the error coefficients of the OVS random error model are calculated and identified by the least squares fitting method, and measures to suppress the random error of OVS are proposed.

\section{RANDOM ERROR CHARACTERISTICS OF OVS}

In practical engineering applications, OVS is not only affected by external environmental factors, including temperature, electromagnetic interference, as well as its own optoelectronic devices aging, mechanical deformation and other factors. OVS output signal contains a series of timevarying noise, there are the shot noise of the photodetector, the fiber thermal phase noise, circuit noise and environmental noise.

These random errors seriously affect the accuracy and reliability of OVS in power system control, protection, measurement and measurement $[5,6]$. There are some relations between the five basic random errors of OVS and the power spectral density $\mathrm{S}_{\Omega}(f)$ of the stochastic process $\Omega(\mathrm{t})$. The specific explanations and corresponding relations are as follows.

In the OVS voltage measurement, the interference signal sampling value from analog to digital converter into a digital input to the central processing unit for signal processing. However, in general, a quantization error occurs because the current phase measured by the OVS is not an integral multiple of the quantization step during the measurement interval.

$$
\begin{gathered}
S_{\Omega}(f) \approx(2 \pi f)^{2} \tau_{0} Q^{2}, f<1 / 2 \tau_{0} \\
\sigma_{Q}^{2}(\tau)=3 Q^{2} / \tau^{2}
\end{gathered}
$$

where $\mathrm{Q}$ is the quantization noise figure. 
Random walk noise voltage is a measure of OVS noise level is an important indicator of accuracy to accurately reflect the limit. This noise is mainly due to shot noise of the photodetector, amplifier noise, electronic device thermal noise and some high-frequency noise with a shorter correlation time than the sampling time. To effectively curb the current random walk, general selection of pure light source, superior performance of photodetectors and other devices, and to maintain the stability of the ambient temperature[7,8]. The noise has a white noise power spectral density of

$$
\begin{gathered}
S_{\Omega}(f)=N^{2} \\
\sigma_{\omega}^{2}(\tau)=N^{2} / \tau
\end{gathered}
$$

where $\mathrm{N}$ is the current random walk coefficient

Bias instability mainly comes from the related discharge components in OVS, circuit noise and environmental noise $[9,10]$. In general, this can be suppressed by improving design stability and a series of filtering methods. Mainly caused by the voltage signal of low-frequency fluctuations. Its rate power spectral density is

$$
\begin{gathered}
S_{\Omega}(f)= \begin{cases}\frac{B^{2}}{2 \pi} \frac{1}{f} & f \leq f_{0} \\
0 & f>f_{0}\end{cases} \\
\sigma_{B}^{2}(\tau) \approx B^{2} \cdot \frac{2}{\pi} \cdot \ln 2
\end{gathered}
$$

where, B is the partial bias instability coefficient, $f_{0}$ is the cutoff frequency.

Random walk at a rate is the result of a power spectral density integral caused by a change in the voltage phase value, which is generally produced by white noise after passing through an integrator and has a long-term effect. The noise rate power spectral density is

$$
\begin{gathered}
S_{\Omega}(f)=\left(\frac{K}{2 \pi}\right)^{2 \cdot} \cdot \frac{1}{f} \\
\sigma_{v}^{2}(\tau)=K^{2} \tau / 3
\end{gathered}
$$

where $\mathrm{K}$ is the rate random walk coefficient.

The rate ramps appear in the OVS input-to-output characteristic data and are generally caused by the extremely slow, monotonic change in intensity of the OVS light source that lasts for a long time, and possibly also from outside temperature changes. Usually, a mathematical model can be set up according to the error compensation method to compensate, so as to reduce the impact of rate ramping noise. The corresponding rate power spectral density is

$$
\begin{gathered}
S_{\Omega}(f)=R^{2} /(2 \pi f)^{3} \\
\sigma_{R}^{2}(\tau)=R^{2} \tau^{2} / 2
\end{gathered}
$$

where $\mathrm{R}$ is the rate ramp coefficient.

Since different types of random errors in OVS occur in different relevant time domains, the power spectral density of different types of noise and related time functions are not the same, so the noise statistics can be independently calculated, and can be obtained synthetically:

$$
\begin{gathered}
\sigma_{\text {Atol }}^{2}=\sigma_{Q}^{2}(\tau)+\sigma_{\omega}^{2}(\tau)+\sigma_{B}^{2}(\tau) \\
+\sigma_{v}^{2}(\tau)+\sigma_{\mathrm{R}}^{2}(\tau) \\
\sigma_{\text {Atol }}^{2}=\frac{R^{2} \tau^{2}}{2}+\frac{K^{2} \tau}{3}+B^{2} \cdot \frac{2}{\pi} \cdot \ln 2 \\
+\frac{N^{2}}{\tau}+\frac{3 Q^{2}}{\tau^{2}}
\end{gathered}
$$

Then, abbreviated as

$$
\sigma_{\text {Atol }}^{2}=\sum_{n=-2}^{2} C_{n} \cdot \tau^{n}
$$

where $\mathrm{Q}$ is the quantization noise coefficient; $\mathrm{N}$ is the current random walk coefficient; $\mathrm{B}$ is the bias bias instability coefficient; $\mathrm{K}$ is the rate random walk coefficient; $\mathrm{R}$ is the rate ramp coefficient.

The least square fitting of the above equation gives the estimated values of the respective random error coefficients.

\section{SEQUENCE ANALYSIS OF VARIANCE}

Let the sample length of the optical voltage transformer drift data be $\mathrm{N}$ and the sampling period be $\mathrm{T}$. The samples are divided into $\mathrm{K}$ groups, each group contains $\mathrm{M}$ sample points, the correlation time of each group is $\tau=\mathrm{M} \cdot \mathrm{T}$, and the raw data is average filtered to obtain a new sample sequence.

$$
K=N / M, M \leq(N-1) / 2
$$

The average for each group is 


$$
\begin{gathered}
\bar{\omega}_{K}(M)=\frac{1}{M} \sum_{i=1}^{M} \bar{\omega}_{(k-1) \cdot M+i} \quad k=1,2,3, \ldots, K \\
\sigma_{A}{ }^{2}\left(\tau_{M}\right)=\frac{1}{2}<\left(\bar{\omega}_{k+1}(M)-\bar{\omega}_{k}(M)\right)^{2} \\
>=\frac{1}{2(k-1)} \sum_{k=1}^{K-1}\left(\bar{\omega}_{k+1}(M)-\bar{\omega}_{k}(M)\right)^{2}
\end{gathered}
$$

The estimation of the sequence variance is based on finite length data and the confidence of the estimate depends on the number of independent groups. For a given random sequence, the smaller the number of groups, the greater the estimation error of the sequence variance $[11,12]$. When the correlation time is long, the estimation of sequence variance fluctuates greatly. At this moment, the variance of sequence has poor estimation confidence, and the error of fitting the sequence variance curve by least square method will cause a big error. In the case of a large averaging factor, data symmetry makes it possible to "collapse" the sequence variance, ie, violent oscillations.

Improved sequential analysis of variance method is a newer tool for frequency domain stability analysis. It extends the OVS signal by the mapping method to improve the degree of freedom of the original data and overcomes the possibility of "collapse" of the variance when the average factor is large The main advantage is that the confidence of the estimate is increased with a large average factor.

Take a set of OVS partial measurement data $\mathrm{x}_{\mathrm{n}}\left(\mathrm{n}=1,2, N_{\mathrm{x}}\right)$, the basic sampling interval is $\tau_{0}$, the total measurement duration $\mathrm{T}=\left(N_{\mathrm{x}}-1\right) \tau_{0}$, the relative frequency offset is $y_{n}=\left(x_{n+1}-x_{n}\right) / \tau_{0}\left(n=1: N_{y}, N_{y}=N_{x}-1\right)$. The sequence $y_{n}$ is extended by mapping into a new, longer virtual sequence. The extending process can be described as

$$
\begin{cases}x_{1-j}^{*}=2 x_{1}-x_{1+j} & \left(j=1, \cdots, N_{y}-1\right) \\ x_{n}^{*}=x_{n} & (n=1,2, \cdots, N) \\ x_{N_{x}+j}^{*}=2 x_{N_{x}}-x_{N_{x}-j} & \left(j=1, \cdots, N_{y}-1\right)\end{cases}
$$

Corresponds to the original time-series $\left\{x_{n}\right\}$, an extended virtual sequence $\left\{x_{n}^{*}\right\}$ is achieved

$$
\begin{cases}x_{1-j}^{*}=2 x_{1}-x_{1+j} & \left(j=1, \cdots, N_{y}-1\right) \\ x_{n}^{*}=x_{n} & (n=1,2, \cdots, N) \\ x_{N_{x}+j}^{*}=2 x_{N_{X}}-x_{N_{x}-j} & \left(j=1, \cdots, N_{y}-1\right)\end{cases}
$$

Inverted-even mapping extends across both ends of the OVS data length to approximately three times as long as the original data, and a dummy data sequence is obtained.

$$
\begin{aligned}
& \operatorname{Im} \operatorname{var}\left(m, N_{x}, \tau_{0}\right)= \\
& \frac{1}{2\left(m \tau_{0}\right)^{2}\left(N_{x}-2\right)} \times \sum_{n=2}^{N_{x}-1}\left(x_{n-m}^{*}-2 x_{n}^{*}+x_{n+m}^{*}\right)^{2}
\end{aligned}
$$

The new variance is obtained by the method of fluctuating the average fractional frequency.

$$
\begin{gathered}
\operatorname{Im} \operatorname{var}\left(m, N_{y}+1, \tau_{0}\right) \\
=\frac{1}{2\left(N_{y}-2\right)} \times \sum_{n=2}^{N_{y}}\left[\bar{y}_{n}^{*}(m)-\bar{y}_{n-m}^{*}(m)\right]^{2} \\
\bar{y}_{n}^{*}=\left(x_{n+m}^{*}-x_{n}^{*}\right) /\left(m \tau_{0}\right)
\end{gathered}
$$

where $\mathrm{m}$ is the average factor, $\tau$ is the total number of samples, $\tau_{0}$ is the basic sampling interval.

The correlation time $\tau$ of the original variance estimation can only be achieved by $N_{y} \tau_{0} / 2$, but the correlation time of the improved variance estimation is reached to $N_{y} \tau_{0}$, and the estimated range is extended by $50 \%$ of the data length.

\section{EXPERIMENT}

To evaluate the random error characteristics of optical voltage sensor, output voltage data of OVS prototype with stable working state was recorded.

The random error characteristics of the model OVS were analyzed by means of sequence variance and modified sequence variance. The test data curve and double logarithmic curve are shown in Fig.1. The blue curve is the sequence variance curve and the red curve is the improved sequence variance curve.

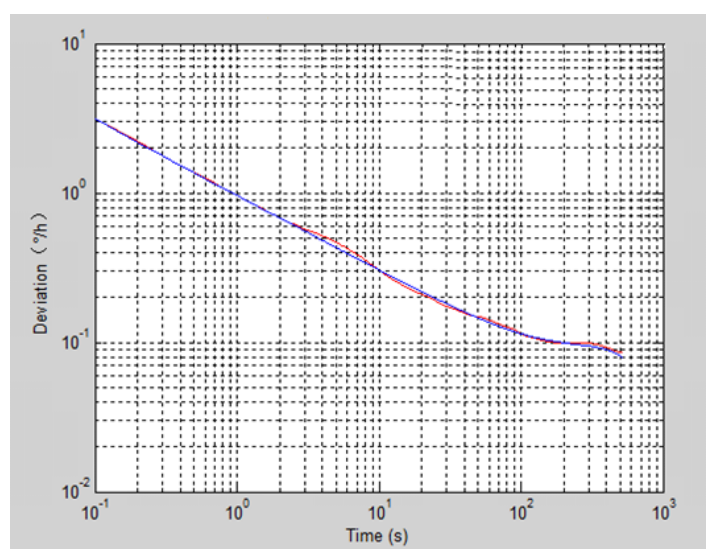

FIGURE I. CURVE OF SEQUENCE VARIANCE AND THE IMPROVED SEQUENCE VARIANCE. 
Figure 1 shows that the sequence variance and the improved sequence variance can be considered as exactly equal when the correlation time is short. However, as the average factor increases, the estimation of the variance of the series tends to fluctuate more and more. Especially at half the total duration of the measurement data, the estimation of the variance of the series changes very rapidly and the estimation error is larger. By improving the sequence variance method, the original data sequence is extended to three degrees of freedom by the inverse mapping method, and the confidence of the variance estimation is improved, so that no oscillation occurs and the oscillation of the sequence variance is solved.

\section{CONCLUSION}

The random error characteristics of optical voltage transformers (OVS) directly affect the measurement accuracy. The sequence variance is a common method to evaluate the random error characteristics of OVS. Aiming at data oscillation problem in the OVS random error by the sequence variance analysis, the improved sequence variance is proposed to analyze the random error characteristics of the OVS. The improved sequence variance method converts the tested timeshifted data into frequency-shifted data and maps the virtual sequences that extend to nearly three times their length for processing. The improved sequence variance method increases the degree of freedom of the data, increases the confidence of the variance estimation, accurately determines the error coefficient of the OVS, and effectively separates the major random error sources. The improved method of sequence variance effectively overcomes the problem of data oscillation when the averaging factor is large, and has obvious advantages in analyzing the random error characteristics of optical voltage transformers.

\section{ACKNOWLEDGMENT}

This work was supported by the following funds: National Natural Science Foundation of China (No. 51477028).

\section{REFERENCES}

[1] Klaus Bohnert, Philippe Gabus, et al, "Theoretical and experimental investigations on the calibration stability of fiber-optic current sensors," Quantum Electronics and Laser Science Conference, pp. 4-5, 2006.

[2] Zhang Xiaohong, Zhu Feng, Xue Xueming, et al, "Using Allan Variance to Analyze the Zero-differenced Stochastic Model Characteristics of GPS," Acta Geodaetica et Cartographica Sinica, vol. 44, pp. 119-127, 2015

[3] Niu Xiaoji, Chen Qijin, Zhang Quan, et al, "Using Allan variance to analyze the error characteristics of GNSS positioning. GPS Solutions," vol. 18, pp. 231-242, 2014.

[4] Shi Guoxiang, Chen Jian, Ye Jun, et al, "Applications of Total Variance Method in Random Error Analysis of the Fiber Optic Gyro Signal," Opto-Electronic Engineering, vol. 39, pp. 708-711, 2007.

[5] Charles A. G, Howe D A, Percival D B, "Total variance, an estimator of long-term frequency stability," IEEE Transactions on Ultrasonic, Ferroelectrics, and Frequency Control, vol. 46, pp. 1183-1191, 1999.

[6] Li, Yansong, Jin Ouyang, Jun Liu, et al, "Analysis on noise of magnetooptical glass type optical current transformer based on Allan variance," Automation of Electric Power Systems, vol. 39, pp. 126-137, 2015.

[7] Tesche, F. M., "On the analysis of a transmission line with nonlinear terminations using the time-dependent BLT equation," IEEE
Transactions on Electromagnetic Compatibility. vol. 49, pp. 427-433, 2007.

[8] Han Junliang, Ge Shengmin, Shen Yi, "Research on the random error properties of FOG based on total variance ," Journal of Harbin Institute of Technology, vol. 39, pp. 08-711, 2007.

[9] Gutierrez M A, Florez J A and Kofuji S T, "Kalman filter and ARMA filter as approach to multiple sensor data fusion problem," 47th International Carnahan Conference on Security Technology (ICCST), vol. 1, pp. 8-11, 2013.

[10] Greenhall C A, Howe D A and Donald B, “ Percival Total variance, an estimator of long-term frequency stability," IEEE Transactions on Ultrasonic, Ferroelectrics, and Frequency Control, vol. 46, pp. 1183$1191,1999$.

[11] Li X, Chen J and Shangguan Y, "A method to analyze and eliminate stochastic noises of FOG based on ARMA and Kalman filtering method," Sixth International Conference on Intelligent Human-Machine System and Cybernetics (IHMSC) vol. 2 325-328, pp. 26-27, 2014.

[12] Jun-Hai Lu, Fan-Bo Meng, Si-Ya Xu, Dong Guo, "A QoS-based ONU Group planning algorithm for smart grid communication network," 19th Asia-Pacific Network Operations and Management Symposium (APNOMS) , pp.25-30, 2017. 